\title{
INFLUENCE OF STRATIFICATION \\ ON THE GROUPS OF CONFORMAL TRANSFORMATIONS OF PSEUDO-RIEMANNIAN ORBIFOLDS
}

\section{N.I. ZHUKOVA}

\begin{abstract}
We study the groups of conformal transformations of $n$-dimensional pseudoRiemannian orbifolds $(\mathcal{N}, g)$ as $n \geqslant 3$. We extend the Alekseevskii method for studying conformal transformation groups of Riemannian manifolds to pseudo-Riemannian orbifolds. We show that a conformal pseudo-Riemannian geometry is induced on each stratum of such orbifold. Due to this, for $k \in\{0,1\} \cup\{3, \ldots, n-1\}$, we obtain exact estimates for the dimensions of the conformal transformation groups of $n$-dimensional pseudo-Riemannian orbifolds admitting $k$-dimensional stratum with essential groups of conformal transforms. A key fact in obtaining these estimates is that each connected transformation group of an orbifold preserves every connected component of each its stratum.

The influence of stratification of $n$-dimensional pseudo-Riemann orbifold to the similarity transformation group of this orbifold is also studied for $n \geqslant 2$.

We prove that the obtained estimates for the dimension of the complete essential groups of conformal transformations and the similarity transformation groups of $n$-dimensional pseudo-Riemann orbifolds are sharp; this is done by adducing corresponding examples of locally flat pseudo-Riemannian orbifolds.
\end{abstract}

Keywords: orbifold, conformal pseudo-Riemannian geometry, conformal transformation, Lie group

Mathematics Subject Classification: 53C50, 53A30

\section{INTRODUCTION}

The study of pseudo-Riemannian manifolds with an essential group of conformal transforms is a topical and actively developing direction of the modern global differential geometry. This is confirmed by the works by Alekseevskii [1], Podoksenov [2], Frances [3]-[4], Frances and Zeghib [5], Frances and Melnick [6] and by other authors as well as by the surveys in monograph [7].

We stress that in distinction to the Riemannian metric, the pseudo-Riemannian metric exists not on each $n$-dimensional orbifold $\mathcal{N}$. Therefore, the existence of a conformal pseudoRiemannian geometry on $\mathcal{N}$ poses conditions on the topology of the orbifold.

The modern theoretical physics employs the geometry on singular stratified spaces, which include also orbifolds. They are used by the physicists as the spaces of strings propagations. The orbifolds turn out to be useful in the conformal field theory. The survey of applications of orbifolds can be found in [8].

N.I. Zhukova, Influence of Stratification on the Groups of CONFORMAL TRANSFORMATIONS OF PSEUDO-RIEMANNIAN ORBIFOLDS.

(C)N.I. Zhukova. 2018.

The manuscript is prepared in the framework of Program "Scientific foundation of National Research University Higher School of Economics" (project no. 16-01-0010) and in the framework of state support for leading universities of Russian Federation "5-100".

Submitted May 8, $201 \%$. 
The orbifolds arise in the foliation theory as "good" spaces of leaves [9]. The known results by Thurston on the classification of closed three-dimensional manifolds use the classification of two-dimensional compact orbifolds [10].

The groups of automorphisms of geometric structures on the orbifolds were studied in [11][13. In [14], there was made the classification of two-dimensional compact Lorentzian orbifolds with non-compact groups of isometries.

The basic facts on orbifolds are described in Section 2, more detailed information can be found in [8].

We consider smooth orbifolds $\mathcal{N}$ of arbitrary dimension $n$ admitting a pseudo-Riemannian metric $g$ of arbitrary signature $(p, q)$, where $p+q=n$. The pair $(\mathcal{N}, g)$ is called pseudoRiemannian orbifold.

Definition 1. Let $\left(\mathcal{N}_{1}, g_{1}\right)$ and $\left(\mathcal{N}_{2}, g_{2}\right)$ be two pseudo-Riemannian orbifolds. A smooth mapping of the orbifolds $f: \mathcal{N}_{1} \rightarrow \mathcal{N}_{2}$ is called conformal if $f^{*} g_{2}=\lambda g_{1}$, where $f^{*}$ is the codifferential of the mapping $f$, and $\lambda$ is a smooth positive function on $\mathcal{N}_{1}$. If $\lambda$ is a constant, then the mapping $f: \mathcal{N}_{1} \rightarrow \mathcal{N}_{2}$ is called a similarity of pseudo-Riemannian orbifolds $\left(\mathcal{N}_{1}, g_{1}\right)$ and $\left(\mathcal{N}_{2}, g_{2}\right)$.

A conformal diffeomorphism of an orbifold $(\mathcal{N}, g)$ onto itself is called a conformal transform. The diffeomorphism of $(\mathcal{N}, g)$ onto itself being a similarity is called a similarity transform.

Definition 2. Two pseudo-Riemannian metrics $g_{1}$ and $g_{2}$ on an orbifold $\mathcal{N}$ are called conformally equivalent (similar) if there exists a smooth positive function (respectively, constant) $\lambda$ on $\mathcal{N}$ such that $g_{2}=\lambda g_{1}$.

A class of conformally equivalent metrics is called a conformal pseudo-Riemannian geometry (or conformal pseudo-Riemannian structure) on $\mathcal{N}$ and is denoted by $[g]$ if the metric $g$ belongs to this class. The class of pseudo-Riemannian metric similar to a metric $g$ is denoted by $[|g|]$.

The group of all conformal transformation of a pseudo-Riemannian orbifold $(\mathcal{N}, g)$ is called a complete group and is denoted by $C(\mathcal{N}, g)$. The group of all similarity transforms of a pseudo-Riemannian orbifold $(\mathcal{N}, g)$ is called a complete group of similarities and is denoted by $\operatorname{Sim}(\mathcal{N}, g)$.

Definition 3. The group of conformal transforms $C(\mathcal{N}, g)$ of a pseudo-Riemannian orbifold is called inessential if it coincides with the group of the isometries of the pseudo-Riemannian orbifold $(\mathcal{N}, h)$, where $h \in[g]$. Otherwise the group $C(\mathcal{N}, g)$ is called essential.

In the same way we define an essential group of similarities of a pseudo-Riemannian orbifold.

The aim of the present work is to study the influence of the stratification of $n$-dimensional pseudo-Riemannian orbifolds on the complete group of its conformal transforms (as $n \geqslant 3$ ) and on its complete group of similarities (as $n \geqslant 2$ ).

We do not consider $C(\mathcal{N}, g)$ in the case $n=2$ since it differs essentially from the conformal geometry as $n \geqslant 3$. For instance, as $n=2$, the conformal group $C O(2)$ is isomorphic to the group $G L(1, \mathbb{C})$ of non-zero complex numbers with the multiplication and each conformal 2-dimensional Riemannian orbifold has a zero conformal curvature.

There exists another equivalent approach to a conformal pseudo-Riemannian geometry based on $G$-structures. Since the conformal pseudo-Riemannian geometry is a second order $G$ structure in the terminology of [17], we can apply Theorem 2 from [11]. Employing this theorem and Theorem VI from [15, Ch. IV], we obtain the following statement.

Theorem 1. For each $n \geqslant 3$, the complete group of conformal transforms $C(\mathcal{N}, g)$ of a $n$ dimensional pseudo-Riemannian orbifold $(\mathcal{N}, g)$ is a Lie group w.r.t. a compactly-open topology and the structure of the Lie group in $C(\mathcal{N}, g)$ is unique and its dimension satisfies the inequality

$$
\operatorname{dim} C(\mathcal{N}, g) \leqslant \frac{(n+1)(n+2)}{2} .
$$


If this group is inessential, then

$$
\operatorname{dim} C(\mathcal{N}, g) \leqslant \frac{n(n+1)}{2} .
$$

Both inequalities become identities only in the case when the orbifold is a manifold, on which the group $C(\mathcal{N}, g)$ acts transitively.

Let $\left(\mathbb{S}^{k}, g_{\mathbb{S}^{k}}\right)$ be a standard $k$-dimensional sphere in the Euclidean space $\mathbb{E}^{k+1}$ with the induced Riemann metric. We consider the product of standard spheres $\mathbb{S}^{p} \times \mathbb{S}^{q}$, where $1 \leqslant p \leqslant q$, equipped by the pseudo-Riemannian metric $-g_{\mathbb{S}^{p}} \oplus g_{\mathbb{S} q}$. The pseudo-Riemmanian manifold $\left(\mathbb{S}^{p} \times \mathbb{S}^{q},-g_{\mathbb{S}^{p}} \oplus g_{\mathbb{S}^{q}}\right)$ is called the Einstein Universe and is denoted by $\operatorname{Ein}^{p, q}$. As it is known, the complete group of conformal transforms of $\operatorname{Ein}^{p, q}$ is essential, is equal to $O(p+1, q+1)$ and has the dimension $\frac{(n+1)(n+2)}{2}$, where $n=p+q$. We observe that under the change of the sign of the metric in $\mathbf{E i n}^{p, q}$, the group of conformal transforms is replaced by the isomorphic Lie group. We stress that the pseudo-Riemannian manifold $\mathbf{E i n}^{p, q}$ is conformally flat. As $n \geqslant 4$, in [4], there was proved the existence of an infinite set of conformal pseudo-Riemannian metric of various signatures on $\mathbb{S}^{1} \times \mathbb{S}^{n-1}$ with essential groups of conformal transform, which are not conformally flat.

The method by Alekseevskii [16] applied him for studying the groups of conformal Riemannian manifolds is extended here to the groups of conformal transforms of pseudo-Riemannian orbifolds. This allows us to obtain the estimates for the dimensions of the group of conformal transforms of a pseudo-Riemannian $n$-dimensional orbifold having a $k$-dimensional stratum, on which an essential group of conformal transforms is induced and to prove the following theorem.

Theorem 2. Let $(\mathcal{N}, g)$ be a n-dimensional, $n \geqslant 3$, pseudo-Riemannian orbifold with a complete essential group of conformal transforms $C(\mathcal{N}, g)$. Then

(i) On each connected component $\Delta_{k}^{c}$ of the $k$-dimensional stratum of the orbifold $\mathcal{N}$ as $k \geqslant 1$, the conformal pseudo-Riemannian structure $\left[\left.g\right|_{\Delta_{k}^{c}}\right]$ is induced.

(ii) If the orbifold $\mathcal{N}$ has the zero-dimensional stratum, then

$$
\operatorname{dim} C(\mathcal{N}, g) \leqslant \frac{n^{2}-n+2}{2} .
$$

(iii) If the orbifold $\mathcal{N}$ has an one-dimensional stratum, then

$$
\operatorname{dim} C(\mathcal{N}, g) \leqslant \frac{n^{2}-3 n+6}{2} .
$$

(iv) If on a $k$-dimensional stratum $\Delta_{k}$ as $3 \leqslant k \leqslant(n-1)$ an essential group of conformal transforms is induced, then

$$
\operatorname{dim} C(\mathcal{N}, g) \leqslant \frac{n(n-1)}{2}+(k+1)^{2}-n k .
$$

There exist orbifolds, for which the estimates in (ii)-(iv) become identities.

Corollary 1. If as $n \geqslant 4$, a n-dimensional pseudo-Riemannina orbifold $(\mathcal{N}, g)$ has a $(n-1)$ dimensional stratum, on which an essential group of conformal transforms is induced, then

$$
\operatorname{dim} C(\mathcal{N}, g) \leqslant \frac{n(n+1)}{2} .
$$

Corollary 2. If a $2 m$-dimensional pseudo-Riemannian orbifold $(\mathcal{N}, g)$ has a $m$-dimensional stratum, $m \geqslant 3$, on which an essential group of conformal transforms is induced, then

$$
\operatorname{dim} C(\mathcal{N}, g) \leqslant m^{2}+m+1
$$


Corollary 3. If a $(2 m-1)$-dimensional pseudo-Riemannian orbifold $(\mathcal{N}, g)$ has a mdimenisonal stratum, $m \geqslant 3$, on which an essential group of conformal transforms is induced, then

$$
\operatorname{dim} C(\mathcal{N}, g) \leqslant m^{2}+2 .
$$

Remark 1. The estimates obtained in Theorem 2 are true for the groups of conformal transforms of Riemannian orbifolds. They improve the estimates obtained in Theorem 5.1 in work [11.

In what follows we study the influence of the stratification of a $n$-dimensional pseudoRiemannian orbifold $(\mathcal{N}, g)$ on the group of $\operatorname{similarities} \operatorname{Sim}(\mathcal{N}, g)$ as $n \geqslant 2$, which is a closed Lie subgroup of the Lie group of the conformal transforms $C(\mathcal{N}, g)$.

Theorem 3. Let $(\mathcal{N}, g)$ be a $n$-dimensional, $n \geqslant 2$, pseudo-Riemannina orbifold with an essential group of similarities $\operatorname{Sim}(\mathcal{N}, g)$. Then

(i) The group $\operatorname{Sim}(\mathcal{N}, g)$ admits the unique structure of the Lie group of the dimension

$$
\operatorname{dim} \operatorname{Sim}(\mathcal{N}, g) \leqslant \frac{n^{2}+n+2}{2} .
$$

(ii) On each connected component $\Delta_{k}^{c}$ of a $k$-dimensional stratum of the orbifold $\mathcal{N}$ with $k \geqslant 1$, a class of similar pseudo-Riemannian metrics $\left[|g|_{\Delta_{k}^{c}} \mid\right]$ is induced.

(iii) If the orbifold $\mathcal{N}$ has the zero-dimensional stratum, then

$$
\operatorname{dim} \operatorname{Sim}(\mathcal{N}, g) \leqslant \frac{n^{2}-n+2}{2} .
$$

(iv) If on a $k$-dimensional stratum $\Delta_{k}$ as $2 \leqslant k \leqslant(n-1)$ an essential group of conformal transforms is induced, then

$$
\operatorname{dim} \operatorname{Sim}(\mathcal{N}, g) \leqslant \frac{n(n-1)}{2}+k^{2}+k+1-n k .
$$

There exist orbifolds, for which estimates (iii) and (iv) become identities.

In Section 7 we construct examples of pseudo-Riemannian orbifolds of arbitrary dimension $n, n \geqslant 3$, for which the estimates in Statements (ii)-(iv) in Theorem 2 and in Statements (iii) and (iv) in Theorem 3 become identities. Estimate (i) in Theorem 3 becomes the identity only in the case of a manifold.

\section{CAtegory of ORBifolds}

Defining an orbifold by an atlas. Let $\mathcal{N}$ be a connected paracompact Hausdorff topological space. Assume that $\Gamma_{U}$ is a finite group of diffeomorphisms of an open connected subset $\widetilde{U}$ in the space $\mathbb{R}^{n}$. We suppose that the group $\Gamma_{U}$ acts effectively on $\widetilde{U}$. We denote by $p_{U}: \widetilde{U} \rightarrow$ $\mathcal{N}$ the composition $q_{U} \circ r_{U}$ of the factor mapping $r_{U}: \widetilde{U} \rightarrow \widetilde{U} / \Gamma_{U}$ and the homeomorphism $q_{U}: \widetilde{U} / \Gamma_{U} \rightarrow U$ on an open subset $U$ of the topological space $\mathcal{N}$. The triple $\left(\widetilde{U}, \Gamma_{U}, p_{U}\right)$ is called the orbifold chart on $\mathcal{N}$ with the coordinate neighbourhood $U$.

We consider two orbifold charts $\left(\widetilde{U}, \Gamma_{U}, p_{U}\right)$ and $\left(\widetilde{V}, \Gamma_{V}, p_{V}\right)$ with neighbourhoods $U$ and $V$, where $U \subset V$. The smooth embedding $\phi_{V U}: \widetilde{U} \rightarrow \widetilde{V}$ obeying $p_{U}=p_{V} \circ \phi_{V U}$ is called the embedding of the chart $\left(\widetilde{U}, \Gamma_{U}, p_{U}\right)$ into the chart $\left(\widetilde{V}, \Gamma_{V}, p_{V}\right)$.

We say that two charts $\left(\widetilde{U}, \Gamma_{U}, p_{U}\right)$ and $\left(\widetilde{V}, \Gamma_{V}, p_{V}\right)$ with coordinate neighbourhoods $U$ and $V$ satisfy the coherent condition if for each point $x \in U \cap V$ there exists a chart $\left(\widetilde{W}, \Gamma_{W}, p_{W}\right)$ with a coordinate neighbourhood $W$ such that $x \in W \subset U \cap V$, for which there exist the embeddings $\phi_{U W}: \widetilde{W} \rightarrow \widetilde{U}$ and $\phi_{V W}: \widetilde{W} \rightarrow \widetilde{V}$. 
Definition 4. The family of the charts $\mathcal{A}=\left\{\left(\widetilde{U}, \Gamma_{U}, p_{U}\right)\right\}$ is called a smooth atlas on $\mathcal{N}$ if it possesses the following two properties:

1) the coordinate neighbourhoods of the charts in $\mathcal{A}$ form an open covering of $\mathcal{N}$;

2) each two charts in $\mathcal{A}$ satisfy the coherent condition.

A connected paracompact Hausdorff topological space $\mathcal{N}$ equipped with a maximal (w.r.t. the inclusion) smooth atlas $\mathcal{A}$ is called an effective $n$-dimensional orbifold and it is still denoted by $\mathcal{N}$.

Smooth mappings of orbifolds. Let $\mathcal{N}$ and $\mathcal{N}^{\prime}$ be smooth orbifolds with atlases $\mathcal{A}$ and $\mathcal{A}^{\prime}$, respectively. A continuous mapping $f: \mathcal{N} \rightarrow \mathcal{N}^{\prime}$ is called smooth if for each point $x \in$ $\mathcal{N}$ there exist charts $\left(\widetilde{U}, \Gamma_{U}, p_{U}\right) \in \mathcal{A}$ and $\left(\widetilde{U}^{\prime}, \Gamma_{U^{\prime}}, p_{U^{\prime}}\right) \in \mathcal{A}^{\prime}$ such that $x \in U=p_{U}(\widetilde{U})$, $f(U) \subset U^{\prime}=p_{U^{\prime}}\left(\widetilde{U^{\prime}}\right)$ and there exists a smooth mapping $f_{U^{\prime} U}: \widetilde{U} \rightarrow \widetilde{U^{\prime}}$ satisfying the identity $p_{U^{\prime}} \circ f_{U^{\prime} U}=\left.f\right|_{U} \circ p_{U}$. The mapping $f_{U^{\prime} U}$ is called the representative of $f$ in charts $\left(\widetilde{U}, \Gamma_{U}, p_{U}\right)$ and $\left(\widetilde{U^{\prime}}, \Gamma_{U^{\prime}}, p_{U^{\prime}}\right)$; at that, $f_{U^{\prime} U}$ is defined up to a composition with the elements in the groups $\Gamma_{U}$ and $\Gamma_{U^{\prime}}$, respectively.

We denote by $\mathfrak{O} \mathfrak{r b}$ the category of orbifolds, whose objects are smooth orbifolds and the morphisms are the smooth mappings of the orbifolds. We note that the category of orbifolds is a complete subcategory in $\mathfrak{O} \mathfrak{r} \mathfrak{b}$.

Pseudo-Riemannian metric on orbifold. To define a pseudo-Riemannian metric $g$ on an orbifold $\mathcal{N}$ means that for each chart $\left(\widetilde{U}, \Gamma_{U}, p_{U}\right)$, on $\widetilde{U}$ a $\Gamma_{U}$-invariant pseudo-Riemannian metric $g_{\widetilde{U}}$ is defined and for each injection $\phi_{V U}: \widetilde{U} \rightarrow \widetilde{V}$ of the chart $\left(\widetilde{U}, \Gamma_{U}, p_{U}\right)$ into the chart $\left(\widetilde{V}, \Gamma_{V}, p_{V}\right)$ the identity holds $\phi_{V U}^{*} g_{\widetilde{V}}=g_{\widetilde{U}}$. The signature of the metric $g_{\widetilde{U}}$ is independent of the choice of a chart in the orbifold atlas and is called the signature of the pseudo-Riemannian metric $g$ on $\mathcal{N}$.

Stratification of orbifolds. For each point $x$ in the orbifold $\mathcal{N}$ there exists a chart $\left(\widetilde{U}, \Gamma_{U}, p_{U}\right)$, whose coordinate neighbourhood $U$ contains $x$. We take $y \in p_{U}^{-1}(x)$ and denote by $\left(\Gamma_{U}\right)_{y}$ the stationary subgroup of the group $\Gamma_{U}$ at the point $y$. We stress that for the given point $x$, an abstract group $\left(\Gamma_{U}\right)_{y}$ is independent of the choice of the chart $\left(\widetilde{U}, \Gamma_{U}, p_{U}\right)$ and $y \in p_{U}^{-1}(x)$. The abstract group $\left(\Gamma_{U}\right)_{y}$ is called the orbifold group of the point $x$ [11]. The point $x$ is called regular if its orbifold group is trivial.

Let $(\mathcal{N}, \mathcal{A})$ be a $n$-dimensional smooth orbifold. We say that points $x$ and $y$ in $\mathcal{N}$ have the same orbifold type if there exist neighbourhoods of these points isomorphic in the category of orbifolds $\mathfrak{O} \mathfrak{r b}$. The subset of the points of the same orbifold type with the induced topology has a natural structure of a smooth manifold, which is, generally speaking, is not connected. The manifolds of points of different type can have the same dimension. We denote by $\Delta_{k}$ the union of the mentioned manifolds of the dimension $k$. There can be $\Delta_{k}=\emptyset$ as $k \in\{0, \ldots, n-1\}$. The family

$$
\Delta(\mathcal{N})=\left\{\Delta_{k} \mid k \in\{0, \ldots, n\}\right\}
$$

is called the stratification of the orbifold $\mathcal{N}$, and $\Delta_{k}$ is called its stratum.

As it is known, see, for instance, [13], the stratification $\Delta(\mathcal{N})=\left\{\Delta_{k} \mid k \in\{0, \ldots, n\}\right\}$ of a $n$-dimensional orbifold $\mathcal{N}$ possesses the following properties.

- Each connected component $\Delta_{k}^{c}$ of the stratum $\Delta_{k}$ is formed by the points of the same orbifold type.

- On the closure $\overline{\Delta_{k}^{c}}$ of the stratum $\Delta_{k}^{c}$, the structure of a $k$-dimensional orbifold is induced, w.r.t. which $\Delta_{k}^{c}$ is the set of regular points.

- The stratum $\Delta_{n}$ formed by regular points is a connected open everywhere dense subset in $\mathcal{N}$. Moreover, $\Delta_{n}$ with an induced smooth structure is a $n$-dimensional manifold. 
We denote by $\operatorname{Diff}(\mathcal{N})$ the group of all diffeomorphisms of the orbifold $\mathcal{N}$. We stress that the stratification of the orbifold $\mathcal{N}$ is invariant w.r.t. the group $\operatorname{Diff}(\mathcal{N})$.

We observe that topological space of a $n$-dimensional orbifold $\mathcal{N}$ as $n \geqslant 3$ is generally speaking not locally Euclidean [12, Example 1].

\section{Proof of Theorem 1}

Choosing an appropriate basis in the pseudo-Euclidean space $\mathbb{E}_{q}^{n}$, we can always reduce the scalar product in this space to the form

$$
g(x, y)=x_{1} y_{1}+\ldots+x_{p} y_{p}-x_{p+1} y_{p+1}-\ldots-x_{n} y_{n},
$$

where $x=\left(x_{1}, \ldots, x_{n}\right), y=\left(y_{1}, \ldots, y_{n}\right) \in \mathbb{E}_{q}^{n}, q+p=n$. The pair of the numbers $(p, q)$ is independent of the choice of the orthonormalized basis of nonisotropic vectors and is called the signature of the pseudo-Euclidean space $\mathbb{E}_{q}^{n}$.

Let $O(p, q)$ be a pseudo-orthogonal group defined by the quadratic form $x_{1}^{2}+\ldots+x_{p}^{2}-$ $x_{p+1}^{2}-\ldots-x_{p+q}^{2}$ of a signature $(p, q)$. The Lie group $C O(p, q)=\mathbb{R}^{+} O(p, q)$, where $\mathbb{R}^{+}$is a multiplicative group of positive number is called a conformal group corresponding to $O(p, q)$.

We let $H:=C O(p, q) \ltimes \mathbb{R}^{n}$, where $n=p+q$ is the semi-direct product of the conformal group $C O(p, q)=R^{+} O(p, q)$ and the normal vector subgroup $\mathbb{R}^{n}$. We regard the Lie group $H$ as the complete group of conformal transforms of the pseudo-Euclidean space $\mathbb{E}_{q}^{n}$. The elements of the group $H$ are denoted by $\left\langle t A, a>\right.$, where $t \in \mathbb{R}^{+}, A \in O(p, q), a \in \mathbb{R}^{n}$. At that, the group operation in $H$ is defined by the identity

$$
<t_{1} A_{1}, a_{1}><t_{2} A_{2}, a_{2}>=<t_{1} t_{2} A_{1} A_{2}, t_{1} A_{1} a_{2}+a_{1}>
$$

for each $<t_{1} A_{1}, a_{1}>,<t_{2} A_{2}, a_{2}>\in H$.

Let $(\mathcal{N},[g])$ be a smooth conformal pseudo-Riemannian orbifold of dimension $n$, where $n \geqslant 3$, and a metric $h \in[g]$ have the signature $(p, q)$.

There exists a natural bijective correspondence between pseudo-Riemannian metrics of signature $(p, q)$ on the orbifold $\mathcal{N}$ and $O(p, q)$-structures on $\mathcal{N}$, that is, defining the conformal geometry $[g]$ on the orbifold $\mathcal{N}$ is equivalent to definining $C O(p, q)$-structure on $\mathcal{N}$. We stress that under the mentioned equivalence, there exists an isomorphism $C(\mathcal{N}, g) \rightarrow \mathfrak{A}$ of the complete group of conformal transforms $C(\mathcal{N}, g)$ and the complete group of automorphisms $\mathfrak{A}$ of the corresponding $C O(p, q)$-structure on $\mathcal{N}$; this diffeomorphism was defined in [11].

As it is known [17], the algebra $\mathfrak{g}=\mathfrak{c o}(p, q)$ of the Lie group $C O(p, q)$ has the order 2 and its first prolongation $\mathfrak{g}_{1}$ is naturally isomoprhic to the dual space $\mathbb{R}^{n *}$ of the vector space $\mathbb{R}^{n}$. Therefore, the $C O(p, q)$-structure is a second order $G$-structure and we can apply the main theorem in [11] to this structure. According this theorem, the complete group of automorphisms $\mathfrak{A} \cong C(\mathcal{N}, g)$ is the Lie group and $\operatorname{dim} C(\mathcal{N}, g)=\operatorname{dim} \mathfrak{A} \leqslant n+\operatorname{dim}\left(\mathfrak{g}+\mathfrak{g}_{1}\right)$. Since $\operatorname{dim} C O(p, q)=$ $\operatorname{dim} \mathfrak{c o}(p, q)=\frac{n(n-1)}{2}+1$, and $\operatorname{dim} \mathfrak{g}_{1}=\operatorname{dim}\left(\mathbb{R}^{n *}\right)=n$, then

$$
\operatorname{dim} C(\mathcal{N}, g)=\operatorname{dim} \mathfrak{A} \leqslant n+\frac{n(n-1)}{2}+1+n=\frac{(n+1)(n+2)}{2} .
$$

We denote by $\mathcal{R}$ the space of $G$-structure on $\mathcal{N}, G=C O(p, q)$. Let $\mathcal{R}^{1}$ be a space of $G_{1^{-}}$ structure on $\mathcal{R}$, where $G_{1}$ is the first prolongation of the group $G$. Let $\pi_{0}: \mathcal{R} \rightarrow \mathcal{N}$ be the $C O(p, q)$ foliation-bundle corresponding to a conformal $G$-structure on $(\mathcal{N}, g)$ and $\pi_{1}: \mathcal{R}^{1} \rightarrow \mathcal{R}$ is the project of the first prolongation of this $G$-structure. Then $\pi=\pi_{0} \circ \pi_{1}: \mathcal{R}^{1} \rightarrow \mathcal{N}$ is the principal $H$-bundle over $\mathcal{N}$, where $H:=C O(p, q) \ltimes \mathbb{R}^{n}$. This means that on the manifold $\mathcal{R}^{1}$, a free smooth right action of the group $H$ is defined such that the space of the orbits $\mathcal{R}^{1} / H$ is the orbifold $\mathcal{N}$, and $\pi$ is a submersion of the orbifolds.

Let $\omega$ be a $\mathfrak{s o}(p+1, q+1)$-valued 1 -form on $\mathcal{R}^{1}$ defining $e$-structure corresponding to the first prolongation of a $G$-structure on $(\mathcal{N}, g)$. Since the pseudo-Riemannian conformal structure on 
$\mathcal{N}$ is a second order $G$-structure, the Lie group of its automorphisms $\mathfrak{A}$ is equal to

$$
\mathfrak{A}=\left\{f \in \operatorname{Diff}\left(\mathcal{R}^{1}\right) \mid f^{*} \omega=\omega, R_{a} \circ f=f \circ R_{a}, a \in H\right\} .
$$

Therefore, $\mathfrak{A}$ is the Lie group of transforms as a closed subgroup of the Lie group of automorphisms of a parallelized manifold $\mathcal{R}^{1}$. This is why, according Theorem VI in [15, Ch. IV], the group $\mathfrak{A}$ is equipped by the compact-open topology and admits the unique structure of the Lie group. Using this, according Proposition 1 in [12], we obtain that in the group $C(\mathcal{N}, g)$ there exists the unique topology and the unique smooth structure, w.r.t. which this is a Lie group and this topology is compact-open.

If the group $C(\mathcal{N}, g)$ is inessential, it coincides with the group of isometries $\operatorname{Iso}(\mathcal{N}, h)$ of some pseudo-Riemannian metric $h \in[g]$. Since the pseudo-Riemannian geometry is a first order $G$-structure, Theorem 2 in [11] yields the estimate

$$
\operatorname{dim} C(\mathcal{N}, g) \leqslant n+\operatorname{dim} O(p, q)=\frac{n(n+1)}{2} .
$$

The last statement of Theorem 1 is implied by [13, Thm. 3.1].

\section{ISOTROPIC REPRESENTATION FOR GROUP OF CONFORMAL TRANSFORMS}

Each connected component $\Delta_{k}^{c}$ of a $k$-dimensional stratum of each orbifold is formed by the points of the same orbifold type and this is why at each point $z \in \Delta_{k}^{c}$ there exists a chart $\left(\widetilde{U}, \Gamma_{U}, p_{U}\right)$ such that all points in $U \cap \Delta_{k}^{c}$ has the same orbifold group $\Gamma_{U}$. Employing this, we obtain the following statement.

Lemma 1. Let $\Delta_{k}^{c}$ be a connected component of a $k$-dimensional stratum of a pseudoRiemannian orbifold $(\mathcal{N}, g)$. Then at each point $z \in \Delta_{k}^{c}$ there exists an orbifold chart $\left(\widetilde{U}, \Gamma_{U}, p_{U}\right)$ possessing the following properties:

1) $\widetilde{U}=\mathbb{R}^{n} \cong \mathbb{R}^{k} \times \mathbb{R}^{n-k}$ and $z=p_{U}(v)$, where $v:=0_{n}$ is the zero in $\mathbb{R}^{n}$;

2) the group $\Gamma_{U}$ is a subgroup of the group $O(p) \times O(q) \subset O(p, q)$;

3) $\mathbb{R}^{k} \times\left\{0_{n-k}\right\}=F i x \Gamma_{U}$ is the set of fixed points of the group $\Gamma_{U}$.

Such chart $\left(\widetilde{U}, \Gamma_{U}, p_{U}\right)$ is called the linearized chart at the point $z$.

Hereafter we employ the linearized $\operatorname{chart}\left(\widetilde{U}, \Gamma_{U}, p_{U}\right)$ at the point $z \in \widetilde{U}$. In the tangent vector space $T_{v} \widetilde{U}$, where $p_{U}(v)=z$, we consider an orthonormalized basis of nonisotropic vectors.

We take any point $z \in \mathcal{N}$ and the $\operatorname{chart}\left(\widetilde{U}, \Gamma_{U}, p_{U}\right)$ at the point $z \in U=p_{U}(\widetilde{U})$. Let $\varphi$ be an arbitrary element of a stationary subgroup $C_{z}(\mathcal{N}, g), z \in \mathcal{N}$ of the complete group of conformal transforms of the pseudo-Riemannian orbifold $(\mathcal{N}, g)$. We consider an arbitrary representative $\bar{\varphi}$ of the transform $\varphi$ in the chart $\left(\widetilde{U}, \Gamma_{U}, p_{U}\right)$. If $v \in \widetilde{U}, p_{U}(v)=z$, then $\bar{\varphi}(v)=v$, that is, $\bar{\varphi} \in C_{v}\left(\widetilde{U}, g_{\widetilde{U}}\right)$.

Since $\bar{\varphi}$ is a conformal transform, that is, there exists a smooth positive $\Gamma_{U}$-invariant function $\lambda$ satisfying the identity $\bar{\varphi}^{*} g_{\widetilde{U}}=e^{\lambda} g_{\widetilde{U}}$. Let $\bar{\varphi}_{* v}=\tau A \in C O(p, q)$ and $\xi=\left.d \lambda\right|_{v}$. We treat $\xi$ as a $n$-dimensional vector. Then the mapping

$$
j: C_{v}\left(\widetilde{U}, g_{\widetilde{U}}\right) \rightarrow H: \varphi \mapsto<\tau A, \xi>
$$

is well-defined. According Theorem 7 in paper [16], we prove the following statement.

Theorem 4. The mapping $j: C_{v}\left(\widetilde{U}, g_{\widetilde{U}}\right) \rightarrow H$ is an isomorphism of the group $C_{v}\left(\widetilde{U}, g_{\widetilde{U}}\right)$ onto the closed subgroup of the group $H$ and for each element $\bar{\varphi}$ in $C_{v}\left(\widetilde{U}, g_{\widetilde{U}}\right)$ the identity $j(\bar{\varphi}) \cdot j\left(\Gamma_{U}\right)=j\left(\Gamma_{U}\right) \cdot j(\bar{\varphi})$ holds.

The mapping $j$ defined in Theorem 4 is called isotropic representation of the group $C_{v}\left(\widetilde{U}, g_{\widetilde{U}}\right)$.

We denote by $E_{k}, k \in \mathbb{N}$, the $k$-dimensional unit matrix. In notation of Theorem 4, we obtain the following statement. 
Corollary 4. Let $z \in \Delta_{k}^{c}$ and $\left(\widetilde{U}, \Gamma_{U}, p_{U}\right)$ is the chart at the point $z$. Then the dimension of the stationary subgroup $C_{z}(\mathcal{N}, g)$ satisfies the inequality

$$
\operatorname{dim} C_{z}(\mathcal{N}, g) \leqslant \operatorname{dim}\left(N\left(\Gamma_{U}\right) / \Gamma_{U}\right) \leqslant \frac{n^{2}-n+2}{2}+k,
$$

where $N\left(\Gamma_{U}\right)$ is the normalizator of the subgroup $\Gamma_{U}$ in the Lie group $H$.

Proof. Since $\Gamma_{U}$ is a finite group, in the linearized group it is realized as a subgroup of the group $O(p, q)$. We identity $\Gamma_{U}$ with $j\left(\Gamma_{U}\right)$ by means of the isomorphism $j$. Let us find the normalizator $N\left(\Gamma_{U}\right)$ in $H$. We observe that $\left\langle\tau A, a>\in N\left(\Gamma_{U}\right)\right.$ if and only if $A$ belongs to the normalizator $N_{0}\left(\Gamma_{U}\right)$ of the group $\Gamma_{U}$ in $C O(p, q)$ and $B a=a$ for each element $<B, 0>\in \Gamma_{U}$. According Lemma 1, $a \in \mathbb{R}^{k}$. Therefore,

$$
\operatorname{dim}\left(N\left(\Gamma_{U}\right) / \Gamma_{U}\right) \leqslant \operatorname{dim} N\left(\Gamma_{U}\right) \leqslant \operatorname{dim} C O(p, q)+k \leqslant \frac{n^{2}-n+2}{2}+k
$$

since $p+q=n$.

In what follows we make us of the following lemma.

Lemma 2. Let $\bar{\varphi}$ be the representative of $\varphi \in C_{z}(\mathcal{N}, g)$ in the chart $\left(\widetilde{U}, \Gamma_{U}, p_{U}\right)$. If $j(\bar{\varphi})=$ $<A, \eta>$, then there exists a $\Gamma_{U}$-invariant metric $h \in[g]$ on $\widetilde{U}$, w.r.t. which the isotropic representation $\bar{\varphi}$ in the same chart is of the form $\langle A, \xi\rangle$, where $A \xi=\xi$.

Proof. We keep the above introduced notations. Assume that $j(\bar{\varphi})=<A, \theta>$ for the representative $\bar{\varphi}$ of the element $\varphi \in C_{z}(\mathcal{N}, g)$ in $\Gamma_{U}$-invariant pseudo-Riemannian metric $g$ on $\widetilde{U}$, where $\bar{\varphi}^{*} g=e^{\lambda} g, \bar{\varphi}_{* v}=A, d \lambda_{v}=\theta$. We stress that $\lambda$ is a $\Gamma_{U}$-invariant function. As it was shown in the proof of Lemma 1 in [16], while passing to another conformal metric $h=e^{\mu} g$ on $\widetilde{U}$, the identity $\bar{\varphi}^{*} h=e^{\beta} h$ holds, where $d \beta_{v}=A \eta-\eta+\theta, \eta=d \mu_{v}$. Denoting $\xi=A \eta-\eta+\theta$, it is easy to show that always there exists a $\Gamma_{U}$-invariant function $\mu$ ensuring the identity $A \xi=\xi$ with $\eta=d \mu_{v}$. At that, the identity holds: $\langle A, \xi>=<E,-\eta><A, \theta><E, \eta>$. Thanks to the $\Gamma_{U}$-invariance of the metric $g$ and the function $\mu$, a new pseudo-Riemannian metric $h=e^{\mu} g$ is $\Gamma_{U}$-invariant.

\section{Proof of Theorem 2}

(i). We begin with proving the following statement.

Lemma 3. On each component of connectivity $\Delta_{k}^{c}$ of a $k$-dimensional stratum $\Delta_{k}$ of a pseudo-Riemannian orbifold $(\mathcal{N}, g)$, a pseudo-Riemannian metric $\left.g\right|_{\Delta_{k}^{c}}$ is induced.

Proof. We consider the linearized chart $\left(\widetilde{U}, \Gamma_{U}, p_{U}\right)$ at an arbitrary point $z \in \Delta_{k}^{c}$. Let $z=p_{U}(v)$, where $v=0_{n}$. We denote $V:=p_{U}\left(\mathbb{R}^{k} \times\left\{0_{n-k}\right\}\right)$. Since the restriction $\left.p_{U}\right|_{\mathbb{R}^{k} \times\left\{0_{n-k}\right\}}: \mathbb{R}^{k} \times$ $\left\{0_{n-k}\right\} \rightarrow V$ is a homeomorphism, the inverse homeomorphism $\psi: V \rightarrow \mathbb{R}^{k} \times\left\{0_{n-k}\right\}$ is welldefined. The pair $(V, \psi)$ is a chart of the manifold $\Delta_{k}^{c}$. We define $g_{V}:=\psi^{*} g_{\widetilde{U}}$. Let us show that $g_{V}$ is a pseudo-Riemannian metric on $V$. The symmetric property of $g_{V}$ is implied by the same of $g_{\widetilde{U}}$. Let us check that $g_{V}$ is non-degenerate. Suppose that there exists a vector $Z \in T_{z} \Delta_{k}^{c}$ such that $g_{V}(X, Z)=0$ for each vector $X \in T_{z} \Delta_{k}^{c}$. It is sufficient to show that $Z=0$.

We denote by $|\Gamma|$ the number of the elements in the group $\Gamma_{U}$. We note that for each vector $Y \in T_{v} \mathbb{R}^{n}$ the vector $\widehat{Y}:=\frac{1}{|\Gamma|} \sum_{\gamma \in \Gamma_{U}} \gamma(Y)$ is stationary w.r.t. each element $\widetilde{\gamma} \in \Gamma_{U}$ and therefore, it belongs to $F i x \Gamma_{U}=\mathbb{R}^{k} \times\left\{0_{n-k}\right\}$, and hence, $p_{U * v} \widehat{Y} \in T_{z} \Delta_{k}^{c}$. Indeed,

$$
\widetilde{\gamma}(\widehat{Y})=\widetilde{\gamma}\left(\frac{1}{|\Gamma|} \sum_{\gamma \in \Gamma_{U}} \gamma(Y)\right)=\frac{1}{|\Gamma|} \sum_{\gamma \in \Gamma_{U}} \widetilde{\gamma} \circ \gamma(Y)=\frac{1}{|\Gamma|} \sum_{\gamma \in \Gamma_{U}} \gamma(Y)=\widehat{Y}
$$


We consider the vectors $\widetilde{Z}:=\psi_{* z} Z$ and an arbitrary vector $Y \in T_{v} \mathbb{R}^{n}$, where $y=\psi(z)$. Applying the linearity of the transforms $\gamma \in \Gamma$, the $\Gamma_{U}$-invariance $g_{\widetilde{U}}$, the stationary property of the vectors $\widehat{Y}$ and $\widetilde{Z}$ w.r.t. $\Gamma_{U}$ as well as the bilinearity of the metric, we obtain the chain of identities

$$
\begin{aligned}
g_{\widetilde{U}}(Y, \widetilde{Z}) & =\frac{1}{|\Gamma|} \sum_{\gamma \in \Gamma_{U}} \gamma^{*} g_{\widetilde{U}}(Y, \widetilde{Z})=\frac{1}{|\Gamma|} \sum_{\gamma \in \Gamma_{U}} g_{\widetilde{U}}(\gamma(Y), \gamma(\widetilde{Z}))=\frac{1}{|\Gamma|} \sum_{\gamma \in \Gamma_{U}} g_{\widetilde{U}}(\gamma(Y), \widetilde{Z}) \\
& =g_{\widetilde{U}}\left(\frac{1}{|\Gamma|} \sum_{\gamma \in \Gamma_{U}} \gamma(Y), \widetilde{Z}\right)=g_{\widetilde{U}}(\widehat{Y}, \widetilde{Z})=g_{V}\left(p_{U * z}(\widehat{Y}), Z\right)=0 .
\end{aligned}
$$

Since the pseudo-Riemannian metric $g_{\widetilde{U}}$ is non-degenerate, this implies $\widetilde{Z}=0$, therefore, $Z=0$ and $g_{V}$ is a pseudo-Riemannian metric.

The consistence of pseudo-Riemannian metrics $g_{V}$ in different charts $(V, \varphi)$ on $\Delta_{k}^{c}$ is implied by the same for the metrics $g_{\widetilde{U}}$ in different charts of the orbifold $\mathcal{N}$.

According Lemma 3, if a pseudo-Riemannian orbifold $(\mathcal{N}, g)$ admits $k$-dimensional stratum $\Delta_{k}$, then on each connected component of this stratum $\Delta_{k}^{c}$, a pseudo-Riemmanian metric $\left.g\right|_{\Delta_{k}^{c}}$ is induced and that transforms it into a pseudo-Riemannian manifold denoted by $\left(\Delta_{k}^{c}, g\right)$. Therefore, the class of conformally equivalent metrics $[g]$ on $\mathcal{N}$ defines a class of conformally equivalent metrics $\left[\left.g\right|_{\Delta_{k}^{c}}\right]$ on $\Delta_{k}^{c}$. Thus, on each stratum of the orbifold a conformal pseudoRiemannian structure is induced. We do not exclude the case when this structure is Riemannian. For instance, on each one-dimensional stratum, if this exists, a conformal Riemannian structure is induced.

(ii). Assume that the orbifold $\mathcal{N}$ has a zero-dimensional stratum $\Delta_{0}$ and $z=\Delta_{0}^{c}$. Since the diffeomorphisms of orbifolds are isomorphisms in the category $\mathfrak{O} \mathfrak{r} \mathfrak{b}$, then each connected Lie group of the diffeomorphisms of orbifolds preserves the components of each stratum. Since the component of the unit $C^{0}(\mathcal{N},[g])$ is a connected Lie group and keeps the point $z$ at its place, Corollary 4 with $k=0$ implies that $\operatorname{dim} C^{0}(\mathcal{N}, g) \leqslant \frac{n^{2}-n+2}{2}$. This completes the proof of Statement (ii).

We consider a pseudo-Riemannian orbifold $(\mathcal{N}, g)$ with a complete group of conformal transforms $C(\mathcal{N}, g)$.

Let $\varphi \in C_{z}(\mathcal{N}, g)$ be a conformal transform, some representative $\bar{\varphi}$ of which has the isotropic representation $\langle A, \xi\rangle$ in the chart $\left(\widetilde{U}, \Gamma_{U}, p_{U}\right)$. According Lemma 1 , without loss of generality, we can assume that $A \xi=\xi$ in the metric $g_{\widetilde{U}}$ in the mentioned class of conformally equivalent metric on $\widetilde{U}$ invariant w.r.t. the group $\Gamma_{U}$. Let $\left(\widetilde{U}, \Gamma_{U}, p_{U}\right)$ be a linearized chart at the point $z \in \mathcal{N}$.

For the sake of brevity, we denote the metric $g_{\widetilde{U}}$ by $g$. We choose a geodesic coordinate system w.r.t the Levi-Civita connection of the pseudo-Riemannian manifold $(\widetilde{U}, g)$. Since we consider only the linearized chart at the point $z$, without loss of generality we assume that the geodesic coordinates are defined in $\widetilde{U}$, where $\widetilde{U}$ is a sufficiently small neighbourhood of zero in $\mathbb{R}^{n}$ invariant w.r.t. the group $\Gamma_{U}$. We recall that $\Gamma_{U}$ is a finite subgroup of a pseudo-orthogonal group $O(p, q)$.

We expand the functions $\bar{\varphi}^{i}$ and $g_{i j}$ into the Taylor series in the geodesic coordinates $x^{i}$ in the vicinity of the point $v=0_{n}$ :

$$
\begin{aligned}
& \bar{\varphi}^{i}(x)=A_{j}^{i} x^{j}+\frac{1}{2} A_{j k}^{i} x^{j} x^{k}+\ldots=A x+\frac{1}{2} A_{x} x+\ldots, \\
& g_{i j}(x)=\delta_{i j}+\frac{1}{2} g_{i j, k l} x^{k} x^{l}+\ldots
\end{aligned}
$$


Since $\bar{\varphi}$ is conformal, we necessarily have $\bar{\varphi}^{*} g=e^{\lambda(x)} g$, therefore, the identity holds

$$
g_{i j}\left(\bar{\varphi}^{k}(x)\right) \frac{\partial \bar{\varphi}^{i}(x)}{\partial x^{m}} \frac{\partial \bar{\varphi}^{j}(x)}{\partial x^{n}}=e^{\lambda(x)} g_{m n}(x) .
$$

We substitute expansions (1) and (2) into (3) and equate the coefficients at the like powers of $x^{i}$. Then, reproducing the arguing in Section 2 in paper [16] by Alekseevskii, we obtain the following expression

$$
\bar{\varphi}(x)=A x+g(x, \xi) A x-\frac{1}{2} g(x, x) \xi+o(g(x, x)) .
$$

Thus, we have proved the following lemma.

Lemma 4. Let $\bar{\varphi}$ be the representative of a conformal transform $\varphi$ from the group $C_{z}(\mathcal{N}, g)$ having the isotropic representation $A \xi=\xi$. Then in terms of geodesic coordinates, the transform $\bar{\varphi}$ can be written as (4) in some neighbourhood of zero.

Let $(\mathcal{N}, g)$ be a pseudo-Riemannian orbifold of dimension $n \geqslant 3$. We denote by $C^{0}(\mathcal{N}, g)$ a component of the unity of the Lie group $C(\mathcal{N}, g)$. Since the dimension of the group $C^{0}(\mathcal{N}, g)$ is equal to the dimension of $C(\mathcal{N}, g)$, in order to estimate the dimension of the group of automorphisms, we consider only the component of the unity $C^{0}(\mathcal{N}, g)$. We assume that $\mathcal{N}$ has a $k$-dimensional stratum as $k \geqslant 3$, and $\Delta_{k}^{c}$ is its connected component. Since each connected Lie group of the diffeomorphisms of the orbifold preserves the components of each stratum, then $C^{0}(\mathcal{N}, g)$ preserves $\Delta_{k}^{c}$.

According Lemma 3 , a pseudo-Riemannian metric is induced on $\Delta_{k}^{c}$; this metric is denoted by $g$. We define the homomorphism of the groups

$$
\chi: C^{0}(\mathcal{N}, g) \rightarrow C\left(\Delta_{k}^{c}, g\right):\left.f \mapsto f\right|_{\Delta_{k}^{c}} .
$$

Then

$$
\operatorname{dim} C(\mathcal{N}, g)=\operatorname{dim} C^{0}(\mathcal{N}, g) \leqslant \operatorname{dim} C\left(\Delta_{k}^{c}, g\right)+\operatorname{dim} \operatorname{Ker}(\chi)
$$

where $\operatorname{Ker}(\chi)$ is the kernel of the homomorphism $\chi$. Employing the estimate for the dimension of the complete group of conformal transforms of a $k$-dimensional manifold $\left(\Delta_{k}^{c}, g\right)$, where $k \geqslant 3$, we obtain

$$
\operatorname{dim} C\left(\Delta_{k}^{c}, g\right) \leqslant \frac{(k+1)(k+2)}{2}
$$

In the next lemma we find an estimate for the dimension of the kernel $\operatorname{Ker}(\chi)$.

Lemma 5. Assume that on a connected component $\Delta_{k}^{c}$ of a $k$-dimensional stratum a pseudoRiemannian metric of type $\left(p_{1}, q_{1}\right)$ is induced, where $p_{1}+q_{1}=k$. Then the kernel Ker $(\chi)$ of the homomorphism

possesses the following properties:

$$
\chi: C^{0}(\mathcal{N}, g) \rightarrow C\left(\Delta_{k}^{c}, g\right):\left.f \mapsto f\right|_{\Delta_{k}^{c}}
$$

1) a representative of each element $f \in K e r \chi$ has an exact isotropic representation in the subgroup $\left\{E_{k}\right\} \times O\left(p-p_{1}, q-q_{1}\right)$ of the pseudo-orthogonal group $O(p, q) \subset H$, where $p+q=n$;

2) the dimension of $\operatorname{Ker}(\chi)$ satisfies the inequality

$$
\operatorname{dim} \operatorname{Ker}(\chi) \leqslant \frac{(n-k)(n-k-1)}{2} .
$$

Proof. We denote by $0_{m}$ the zero in $\mathbb{R}^{m}, m \in \mathbb{N}$. We take an element $\varphi \in \operatorname{Ker}(\chi)$, then $\left.\varphi\right|_{\Delta_{k}^{c}}=i d_{\Delta_{k}^{c}}$. In the linearized chart $\left(\widetilde{U}, \Gamma_{U}, p_{U}\right)$ at an arbitrary point $z \in \Delta_{k}^{c}$, the representative $\bar{\varphi}$ of the transform $\varphi$ satisfies the identity $\left.\bar{\varphi}\right|_{\mathbb{R}^{k} \times\left\{0_{n-k}\right\}}=i d_{\mathbb{R}^{k} \times\left\{0_{n-k}\right\}}$, where $p_{U}(0,0)=z,(0,0)=$ $\left(0_{k}, 0_{n-k}\right)=0_{n}$. We identify the tangent space $T_{(0,0)}\left(\mathbb{R}^{k} \times\left\{0_{n-k}\right\}\right)$ with $\mathbb{R}^{k} \times\left\{0_{n-k}\right\}$, and $T_{(0,0)}\left(\left\{0_{k}\right\} \times \mathbb{R}^{n-k}\right)$ is identified with $\left\{0_{k}\right\} \times \mathbb{R}^{n-k}$. According Lemma 4 , on $\mathbb{R}^{k} \times\left\{0_{n-k}\right\}$ a pseudo-Riemannian metric is induced. Without loss of generality, we suppose that $\left\{0_{k}\right\} \times \mathbb{R}^{n-k}$ 
is the orthogonal complement to $\mathbb{R}^{k} \times\left\{0_{n-k}\right\}$. Since the conformal transforms preserve the angles between the vectors, $\left\{0_{k}\right\} \times \mathbb{R}^{n-k}$ is invariant w.r.t. $\bar{\varphi}_{*(0,0)}$.

We assume that $\bar{\varphi}_{*(0,0)}=\tau A \in C O(p, q)$. Since $\varphi \in \operatorname{Ker}(\chi)$, the identity $\bar{\varphi}_{*(0,0)}(e)=\tau A(e)=$ $e$ holds for each vector $e \in \mathbb{R}^{k} \times\left\{0_{n-k}\right\}$. Therefore, $\tau=1$ and $A \in\left\{E_{k}\right\} \times O\left(p-p_{1}, q-q_{1}\right)$. Thus, $\bar{\varphi}$ has the isotropic representation $j(\bar{\varphi})=<A, \xi>$. Without loss of generality, by Lemma 2 we can assume that $A \xi=\xi$. Hence, according Lemma 4, in terms of the geodesic coordinates, in some neighbourhood $\widetilde{U}$ of zero, the representative $\bar{\varphi}$ of the element $\varphi$ satisfies equation (4).

Let $e_{i}, 1 \leqslant i \leqslant k$, be a basis in the vector space $\mathbb{R}^{k} \times\left\{0_{n-k}\right\}$ of nonisotropic vectors. Since $A \in\left\{E_{k}\right\} \times O\left(p-p_{1}, q-q_{1}\right)$, we necessarily have $A x=x$ for each vector $x$ in $\mathbb{R}^{k} \times\left\{0_{n-k}\right\}$. We take an arbitrary infinitesimal real non-zero number $\alpha$ and substitute $x=\alpha e_{i}$ into (4). Taking into consideration that $A e_{i}=e_{i}$, we obtain

$$
\alpha\left[\alpha g\left(e_{i}, \xi\right) e_{i}-\frac{\alpha}{2} g\left(e_{i}, e_{i}\right) \xi+o\left(\alpha g\left(e_{i} e_{i}\right)\right)\right]=0
$$

and this is equivalent to

$$
g\left(e_{i}, \xi\right) e_{i}-\frac{1}{2} g\left(e_{i}, e_{i}\right) \xi+\frac{o\left(\alpha g\left(e_{i} e_{i}\right)\right)}{\alpha}=0 .
$$

Since $\frac{o\left(\alpha g\left(e_{i} e_{i}\right)\right)}{\alpha} \rightarrow 0$ as $\alpha \rightarrow 0$, it follows from 5 that

$$
g\left(e_{i}, \xi\right) e_{i}=\frac{1}{2} g\left(e_{i}, e_{i}\right) \xi
$$

By the choice of the basis $e_{i}$ we have $g\left(e_{i}, e_{i}\right) \neq 0$ and this is it follows from (6) that the vectors $\xi$ and $e_{i}$ are collinear. In this case $\xi=c e_{i}$. Substituting this into (6), we obtain $c=0$ and $\xi=0$

Thus, we have proved that $\varphi \in \operatorname{Ker}(\chi)$ has the isotropic representation of form $\langle A, 0\rangle$, where $A \in\left\{E_{k}\right\} \times O\left(p-p_{1}, q-q_{1}\right)$. This implies Statement 1) and the desired estimate 2).

Applying Lemmata 2 and 4 , we obtain the chain of inequalities

$$
\begin{aligned}
\operatorname{dim} C(\mathcal{N}, g) & \leqslant \operatorname{dim} C\left(\Delta_{k}^{c}, g\right)+\operatorname{dim} \operatorname{Ker}(\chi) \\
& \leqslant \frac{(k+1)(k+2)}{2}+\frac{(n-k)(n-k-1)}{2}=\frac{n(n-1)}{2}+(k+1)^{2}-n k
\end{aligned}
$$

and this completes the proof of Statement (iv) of Theorem 2 .

Statement (iii) of Theorem 2 as $k=1$ can be proved in the same way as Statement (iv) by using the inequality $\operatorname{dim}\left(C\left(\Delta_{1}^{c}, g\right)\right) \leqslant 2$.

\section{Groups of Similarities of PSEUdo-Riemannian orbifolds}

Proof of Theorem 3. Since each similarity is a conformal transform and $\operatorname{Sim}(\mathcal{N}, g)$ is a closed subgroup of the Lie group $C(\mathcal{N}, g)$, all facts proved for the groups of conformal transforms of pseudo-Riemannian orbifolds are true for the groups of similarities. This is why there exists the unique structure of the Lie group in $\operatorname{Sim}(\mathcal{N}, g)$. Since $\operatorname{Sim}(\mathcal{N}, g)$ is the group of automorphisms of first order $G$-structure in the terminology [17], by [11] we get the estimate

$$
\operatorname{dim}(\operatorname{Sim}(\mathcal{N}, g)) \leqslant n+\operatorname{dim} C O(p, q)=n+\frac{n^{2}-n+2}{2}=\frac{n^{2}+n+2}{2},
$$

and here the identity can be only in the case of a transitive action of group of similarities on $\mathcal{N}$, that is, only for manifolds. Thus, Statement (i) is true.

Statement (ii) can be easily obtained by applying Lemma 3

We consider an arbitrary similarity transform $\varphi \in \operatorname{Sim}_{z}(\mathcal{N}, g)$ preserving some point $z \in \mathcal{N}$ at its place. Let $\bar{\varphi}$ be a representative of $\varphi$ in some chart $\left(\widetilde{U}, \Gamma_{U}, p_{U}\right)$ of the orbifold at the point $z$. Then there exists a positive number $\lambda$ satisfying the identity $\bar{\varphi}^{*} g=e^{\lambda} g$. Let $\bar{\varphi}_{* z}=$ 
$\tau A \in C O(p, q)$. We stress that in this case $\xi=\left.d \lambda\right|_{z}=0$. It follows from Theorem 4 that the representation of the isotropy for $\bar{\varphi}$ is of the form:

$$
j: \operatorname{Sim}_{v}(\widetilde{U}, g) \rightarrow H=C O(p, q): \bar{\varphi} \mapsto<\tau A, 0>,
$$

where $v \in p_{U}^{-1}(z)$, and $j$ is an isomorphism of the group $\operatorname{Sim}_{v}(\widetilde{U},[g])$ onto a closed subgroup of the group $H=C O(p, q)$. Taking into consideration that $\operatorname{dim}(H)=\frac{n^{2}-n+2}{2}$ and reproducing the arguing in the proofs of Statements (ii) and (iv) of Theorem 2, we obtain the estimate (iii) and (iv).

\section{EXAMPLES}

We recall that the $n$-dimensional pseudo-Euclidean space $\mathbb{E}_{q}^{n}$ of signature $(p, q), p+q=n$, with the standard pseudo-Riemannian metric $g_{0}$ defines a conformal structure $\left[g_{0}\right]$ corresponding to the first order $G$-structure. This is why the complete group of conformal transforms $C\left(\mathbb{E}_{q}^{n}, g_{0}\right)$ coincides with the complete group of similarities $\operatorname{Sim}\left(\mathbb{E}_{q}^{n}, g_{0}\right)$ and is equal to the semi-direct product $C O(p, q) \ltimes \mathbb{R}^{n}$.

Example 1. According the definition introduced in Section 3 , the signature of $\mathbf{E i n}^{p, q}$ is equal to $(q, p)$. The stationary subgroup $C_{v}\left(\mathbf{E i n}^{p, q}\right)$ of the group of conformal transforms $C\left(\operatorname{Ein}^{p, q}\right)$ at the point $v$ is isomorphic to the group $H=C O(p, q) \ltimes \mathbb{R}^{n}$. According Theorem 4, the represenation of isotropy $j: C_{v}\left(\mathbf{E i n}^{p, q}\right) \rightarrow H$ is an isomorphism of the groups. Therefore, the conformal transform $\gamma \in C_{v}\left(\mathbf{E i n}^{p, q}\right)$ is uniquely determined by its image $j(\gamma)=\left(-E_{n}\right) \in H$, where $E_{n}$ is the unit $n$-dimensional matrix. We consider the orbifold $\mathcal{N}=\operatorname{Ein}^{p, q} / \Gamma$, where $\Gamma$ is the group with the generator $\gamma$ and $\Gamma$ is isomorphic to $\mathbb{Z}_{2}$. We denote by $g_{0}$ the induced metric on $\mathcal{N}$. The Lorentzian orbifold $\left(\mathcal{N}, g_{0}\right)$ has the stratification $\left\{\Delta_{n}, \Delta_{0}\right\}$. Let $r: \operatorname{Ein}^{p, q} \rightarrow \mathcal{N}$ be a factor mapping and $z=r(v)$. Since the group of conformal transforms $C\left(\mathcal{N}, g_{0}\right)$ preserves the stratification of the orbifold $\mathcal{N}$, it coincides with the stationary subgroup $C_{z}\left(\mathcal{N}, g_{0}\right)$. Taking this fact into consideration and applying Corollary 4, we obtain that the group of conformal transforms $C\left(\mathcal{N}, g_{0}\right)$ of the Lorentzian orbifold $\left(\mathcal{N}, g_{0}\right)$ is isomorphic to the factor group $N(\Gamma) / \Gamma$, where $N(\Gamma)$ is the normalizator of the subgroup $\Gamma$ in the Lie group $H$. By straightforward calculations we check that $N(\Gamma)=C O(p, q)$. Since

$$
\operatorname{dim}\left(\mathbb{R}^{+} O(p, q) /\left\{ \pm E_{n}\right\}\right)=\frac{n^{2}-n+2}{2},
$$

then $\operatorname{dim}\left(C\left(\mathcal{N}, g_{0}\right)\right)=\left(n^{2}-n+2\right) / 2$ and the estimate for the dimension of the conformal group $C\left(\mathcal{N}, g_{0}\right)$ in Statement (ii) of Theorem 2 becomes the identity.

Example 2. We consider the orbifold $\mathcal{N}=\mathbb{E}_{q}^{n} / \Gamma$, where $\Gamma$ is the group isomorphic to $\mathbb{Z}_{2}$ and having the generator $\gamma$ defined by the identity $\gamma(x)=-x, x \in \mathbb{E}_{q}^{n}$. We denote by $g_{0}$ the induced pseudo-Riemannian metric on $\mathcal{N}$. Then the pseudo-Euclidean orbifold $\left(\mathcal{N}, g_{0}\right)$ has the stratification $\left\{\Delta_{n}, \Delta_{0}\right\}$ and the complete group of its conformal transforms $C\left(\mathcal{N}, g_{0}\right)$ coincides with the complete group of $\operatorname{similarities} \operatorname{Sim}\left(\mathcal{N}, g_{0}\right)$ and is equal to the factor group $\mathbb{R}^{+} O(p, q) /\left\{ \pm E_{n}\right\}$ and this is why $\operatorname{dim}\left(C\left(\mathcal{N}, g_{0}\right)\right)=\left(n^{2}-n+2\right) / 2$. Hence, the estimate for the dimension of the complete group of similarities $\operatorname{Sim}\left(\mathcal{N}, g_{0}\right)$ in Statement (iii) in Theorem 3 becomes the identity.

Example 3. We recall that according the definition introduced in Section 3 , the signature of $\operatorname{Ein}^{q_{1}, p_{1}}$ is equal to $\left(p_{1}, q_{1}\right)$. Let $p_{1}+q_{1}=k \geqslant 3$. We consider the orbifold $\mathcal{N}_{1}=\mathbb{E}_{q_{2}}^{n-k} / \Gamma$ considered in Example 2. It is of the signature $\left(p_{2}, q_{2}\right)$, where $p_{2}+q_{2}=n-k \geqslant 2$. Let $\left\{\Delta_{n-k}^{(1)}, \Delta_{0}^{(1)}\right\}$ be the stratification of $\mathcal{N}_{1}$.

Let us calculate the complete group of conformal transforms of the pseudo-Riemannian orbifold $\mathcal{N}=\operatorname{Ein}^{q_{1}, p_{1}} \times \mathcal{N}_{1}$ with the metric $g$ equal to the sum of the corresponding metrics. We 
note that $\mathcal{N}$ has the stratification $\left\{\Delta_{n}, \Delta_{k}\right\}$ and $\Delta_{k}=\operatorname{Ein}^{q_{1}, p_{1}} \times \Delta_{0}^{(1)}$. Since the group $C(\mathcal{N}, g)$ preserves the connected stratum $\Delta_{k}$, by Lemma 5 we obtain that the Lie group $C(\mathcal{N}, g)$ is isomorphic to the product of the Lie groups $C\left(\mathbf{E i n}^{q_{1}, p_{1}}\right) \times O\left(p_{2}, q_{2}\right)$. Therefore, the chain of identities hold:

$$
\operatorname{dim} C(\mathcal{N}, g)=\operatorname{dim} C\left(\mathbf{E i n}^{q_{1}, p_{1}}\right)+\operatorname{dim} O\left(p_{2}, q_{2}\right)=\operatorname{dim} O\left(q_{1}+1, p_{1}+1\right)+\operatorname{dim} O\left(p_{2}, q_{2}\right),
$$

and this is why

$$
\operatorname{dim} C(\mathcal{N}, g)=\frac{(k+1)(k+2)}{2}+\frac{(n-k)(n-k-1)}{2}=\frac{n(n-1)}{2}+(k+1)^{2}-n k .
$$

Thus, the estimate for the dimension of the group $C(\mathcal{N}, g)$ in Statement (iv) of Theorem 2 becomes the identity.

Remark 2. For the group of similarities $\operatorname{Sim}(\mathcal{N}, g)$ the statement of Lemma 5 is true for the connected component $\Delta_{k}^{0}$ of a $k$-dimensional stratum for each fixed $k \geqslant 1$.

Example 4. We fix an arbitrary pair of natural numbers $(n, k)$ such that $n>k \geqslant 1$. Let $\left(\mathcal{N}_{1}, g_{0}\right)$ be a pseudo-Euclidean orbifold constructed in Example 2 of dimension $n-k$ and with a signature $\left(p_{1}, q_{1}\right)$, where $p_{1}+q_{1}=n-k$. The orbifold $\mathcal{N}_{1}$ has the stratification $\left\{\Delta_{n-k}^{(1)}, \Delta_{0}^{(1)}\right\}$. Let $\left(\mathbb{E}_{q_{2}}^{k}, g_{2}\right)$ be a pseudo-Euclidean space of dimension $k$ and with a signature $\left(p_{2}, q_{2}\right)$, where $p_{2}+q_{2}=k$. The product of the orbifolds $\mathcal{N}:=\mathcal{N}_{1} \times \mathbb{E}_{q_{2}}^{k}$ with the metric $g:=g_{0} \oplus g_{2}$ is a $n$-dimensional pseudo-Euclidean orbifold with a signature $(p, q), p+q=n$, where $p=p_{1}+p_{2}$, $q=q_{1}+q_{2}$. The orbifold $\mathcal{N}$ has the stratification $\left\{\Delta_{n}, \Delta_{k}=\Delta_{0}^{(1)} \times \mathbb{E}_{q_{2}}^{k}\right\}$. We observe that the complete group of conformal transforms $C(\mathcal{N}, g)$ coincides with the complete group of similarities $\operatorname{Sim}(\mathcal{N}, g)$. Since the $\operatorname{group} \operatorname{Sim}(\mathcal{N}, g)$ preserves the connected stratum $\Delta_{k}$, in view of Lemma 5 and Remark 2, we obtain the existence of the isomorphism between the Lie $\operatorname{group} \operatorname{Sim}(\mathcal{N}, g)$ and the product of the Lie groups $\operatorname{Sim}\left(\mathbb{E}_{q_{2}}^{k}, g_{2}\right) \times O\left(p_{1}, q_{1}\right)$. Therefore, the chain of identities holds:

$$
\begin{aligned}
\operatorname{dim} \operatorname{Sim}(\mathcal{N}, g) & =\operatorname{dim} \operatorname{Sim}\left(\mathbb{E}_{q_{2}}^{k}, g_{2}\right)+\operatorname{dim} O\left(p_{1}, q_{1}\right) \\
& =\frac{k^{2}+k+2}{2}+\frac{(n-k)(n-k-1)}{2}=\frac{n(n-1)}{2}+k^{2}+k+1-n k,
\end{aligned}
$$

and this is why

$$
\operatorname{dim} \operatorname{Sim}(\mathcal{N}, g)=\frac{n(n-1)}{2}+k^{2}+k+1-n k .
$$

Thus, in Statement (iii) in Theorem 3 we have the identity.

Let $k=1$, then by the above identity we obtain

$$
\operatorname{dim}(C(\mathcal{N}, g))=\frac{n^{2}-3 n+6}{2}
$$

and the estimate for the dimension of the complete group of similarities $C(\mathcal{N}, g)$ in Statement (iii) of Theorem 2 becomes the identity.

\section{BIBLIOGRAPHY}

1. D.V. Alekseevskii. Lorentzian manifolds with transitive conformal group // Note di Matematica. 37:Suppl. 1, 35-48 (2017).

2. M.N. Podoksenov. A Lorentzian manifold with a group of conformal transformations that contains a normal one-parameter subgroup of homotheties // Sibir. Matem. Zhurn. 38:6, 1356-1359 (1997). [Siber. Math. J. 38:6, 1178-1181 (1997).]

3. C. Frances Sur les variétés lorentziennes dont le group conforme est essentiel // Math. Ann. 332:1, 103-119 (2005). 
4. C. Frances. About pseudo-Riemannian Lichnerovitcz conjecture // Transformation Groups. 29:4, 1015-1022 (2015).

5. C. Frances, A. Zeghib. Some remarks on pseudo-Riemannian conformal actions of simple Lie groups // Math. Res. Lett. 12:1, 49-56 (2005).

6. C. Frances, K. Melnick. Conformal actions of nilpotent groups on pseudo-Riemannian manifolds // Duke Math J. 153:3, 511-550 (2010).

7. V. Alekseevsky, H. Baom. Resent development in pseudo-Riemannian geometry. ESI Lect. Math. Phys. Eur. Math. Soc., Zurich (2008).

8. A. Adem, J. Leida and Y. Ruan. Orbifolds and stringy topology. Cambridge Tracts in Mathematics. 171, Cambridge Univ. Press, New York (2007).

9. N.I. Zhukova. Local and global stability of compact leaves and foliations // J. Math. Phys. Anal. Geom. 9:3, 400-420 (2013).

10. W.P. Thurston. The geometry and topology of 3-manifolds. Princeton Univ. Press, Princeton (1978).

11. A.V. Bagaev, N.I. Zhukova. The automorphism groups of finite type G-structures on orbifolds // Sibir. Matem. Zhurn. 44:2, 263-278 (2003). [Siber. Math. J. 44:2, 213-224 (2003).]

12. A.V. Bagaev, N.I. Zhukova. The isometry groups of Riemannian orbifolds // Sibir. Matem. Zhurn. 48:4, 723-741 (2007). [Siber. Math. J. 48:4, 579-592 (2007). ]

13. A.V. Bagaev, N.I. Zhukova. The automorphism group of some geometric structures on orbifolds // In "Lie groups: New Research", Nova Science Publishers, New York, Ch. 16, 447-483 (2009).

14. N.I. Zhukova, E.A. Rogozhkina. Classification of compact Lorentzian 2-orbifolds with noncompact full isometry groups // Sibir. Matem. Zhurn. 53:6, 1292-1309 (2012). [Siber. Math. J. 53:6, 1037-1050 (2012).]

15. R.S. Palais. A global formulation of the Lie theory of transformation groups // Mem. Amer. Math. Soc. 22, 1-129 (1957).

16. D.V. Alekseevskii. Groups of conformal transformations of Riemannian spaces // Matem. Sborn. 18:2, 280-296 (1972). [Math. USSR-Sb. 18:2, 285-301 (1972).]

17. S. Kobayashi. Transformation groups in differential geometry. Springer-Verlag, New York (1995).

Nina Ivanovna Zhukova,

National Research University

Higher School of Economics,

Bol'shaya Pecherskaya str. 25/12,

603155, Nizhny Novgorod, Russia

E-mail: nzhukova@hse.ru 